\title{
Transatlantica
}

Revue d'études américaines. American Studies Journal

$1 \mid 2002$

Jeune République

\section{" Agriculture, and Commerce as its Handmaid »}

L'économie politique et les toasts publics en 1793

\section{Pierre Gervais}

\section{CpenEdition}

Journals

Édition électronique

URL : http://journals.openedition.org/transatlantica/463

DOI : 10.4000/transatlantica.463

ISSN : $1765-2766$

Éditeur

AFEA

Référence électronique

Pierre Gervais, « « Agriculture, and Commerce as its Handmaid » », Transatlantica [En ligne], 1 | 2002, mis en ligne le 23 mars 2006, consulté le 29 avril 2021. URL : http://journals.openedition.org/ transatlantica/463 ; DOI : https://doi.org/10.4000/transatlantica.463

Ce document a été généré automatiquement le 29 avril 2021

\section{(c) $($ () $\ominus$}

Transatlantica - Revue d'études américaines est mis à disposition selon les termes de la licence Creative Commons Attribution - Pas d'Utilisation Commerciale - Pas de Modification 4.0 International. 


\section{« Agriculture, and Commerce as its Handmaid »}

L'économie politique et les toasts publics en 1793

Pierre Gervais

1 L'idéologie jeffersonienne et son opposée fédéraliste ont fait couler tant d'encre qu'il paraît presque présomptueux de vouloir rouvrir le débat. Les sources ont été disséquées, en remontant jusqu'aux révolutions anglaises $\mathrm{du} \mathrm{xvII}^{\mathrm{e}}$ siècle, sans omettre le moindre auteur que Jefferson ou Hamilton auraient pu avoir lu. Le caractère réel ou non de l'opposition entre les deux parties dans un cadre général de nationalisme exacerbé et de "religion civique" a soulevé d'interminables controverses. Le radicalisme de Jefferson a été affirmé, puis nié, puis réaffirmé ; le pragmatisme de Hamilton a subi le même sort. Les innovations des Pères Fondateurs en science politique, l'apparition de l'idée de parti d'opposition, le concept d'équilibre entre factions rivales, ont été abondamment soulignés, introduisant dans l'opposition entre Jefferson et Hamilton un facteur de complication supplémentaire, la théorie politique madisonienne. Toutes ces discussions ont d'ailleurs souvent abouti à des descriptions curieusement vides de différends sur l'économie, les analyses historiques mettant l'accent sur diverses notions politiques, vertu, république, rôle des partis, ou équilibre entre pouvoir et liberté, plutôt que sur les luttes d'intérêt. Même les thèses de Joyce Appleby et de John R. Nelson, qui semblaient réintroduire une dimension économique dans les pensées jeffersoniennes et hamiltoniennes, les présentent en dernière analyse comme deux faces tactiquement différentes d'une même stratégie dominée par le libéralisme des Lumières, avec un consensus entier sur les principes : propriété privée, libre marché, esprit commercial, volonté de développement économique ${ }^{1}$. Nul ne nie que les positions adoptées de part et d'autre avaient des conséquences concrètes, sur les finances publiques, le rôle de l'État, l'évolution économique et sociale jugée la plus souhaitable et la plus propre à sauvegarder la république. Mais ces conséquences ne seraient que cela, des conséquences, découlant de visions opposées du fonctionnement politique lui-même, dans l'évaluation du rôle respectif du peuple et des élites, ou au 
plus dans le choix des groupes sur lesquels devrait s'appuyer en priorité le développement de la nouvelle nation ${ }^{2}$.

2 Le développement récent d'un consensus « libéral » pose cependant un problème, déjà présent dans l'historiographie "républicaine ": que faire du torrent de rhétorique agrarienne qui envahissait chaque déclaration des démocrates-républicains ? Une réponse possible est qu'il ne s'agissait justement que de rhétorique, sans contenu particulier. A la lecture de la presse des années 1790, et des essais des principaux responsables, Madison, Jefferson, Hamilton et autres, pareille réponse laisse un peu le chercheur sur sa faim. S'il ne s'agissait véritablement que de rhétorique, pourquoi a-t-elle pris tant d'importance, au point de dominer la tradition démocrate, de structurer la pensée Populiste un siècle plus tard, et même, en un ultime avatar avant la grande modernisation de la gauche américaine, d'occuper une place centrale dans le premier discours inaugural de Franklin Roosevelt (Peterson 1960) ? L'opposition entre agriculteurs vertueux et «hommes d'argent" corrompus et anglophiles, caractéristique de tous les discours jeffersoniens des années 1790, qu'ils soient publics ou privés, est pourtant systématiquement formulée en termes économiques : c'est la position économique qui fonde l'attitude politique, et non l'inverse. L'historien se trouve donc face à un choix délicat. On peut choisir de considérer comme des effets rhétoriques, et donc de minimiser, les déclarations pourtant aussi nombreuses qu'explicites de responsables politiques de premier plan, ce qui est une solution peu satisfaisante. L'autre terme de l'alternative est de tenter de réconcilier ce discours socio-politique conflictuel avec l'accord qui semblait exister entre toutes les parties sur les grands principes de l'économie libérale ; c'est ce que nous tenterons de faire ici ${ }^{3}$. L'article qui suit n'est cependant qu'un premier essai en ce sens, puisqu'il s'agit d'une recherche en cours : ces premières pistes devront être approfondies, en particulier en élargissant la gamme de textes et de sources sur lesquelles s'appuie mon analyse. A ce stade, et à partir de sources restreintes, il me semble tout de même possible de proposer une nouvelle manière de replacer le conflit économique au centre du conflit sur l'économie politique, en partant de quelques prises de position de Jefferson et Hamilton eux-mêmes sur le sujet, et en montrant que ces positions étaient au cœur, et non à la périphérie, de leur discours politique. Dans une seconde partie, j'analyserai un échantillon également restreint de discours politique "publics", constitué par des « toasts " portés à diverses occasions pendant l'année 1793, et montrerai que les mêmes lignes de force déjà identifiées chez les deux principaux chefs de parti de la période se retrouvent dans ces énonciations.

1. «Economie » et « économie politique » : Jefferson, Hamilton, et les conflits économiquesA) Jefferson et les moneyed men

3 En 1785, dans les Notes on the State of Virginia, Jefferson affirmait clairement son opposition de principe à tout développement commercial générateur de puissance marchande : «Corruption of morals [...] is the mark set on those, who, not looking up to heaven, to their own soil and industry, as does the husbandman, for their subsistence, depend for it on the casualties and caprices of customers. Dependence begets subservience and venality, suffocates the germ of virtue, and prepares fit tools for the designs of ambition ${ }^{4} »$. Ce passage bien connu est parfois compris comme un appel à l'autarcie, ce qu'il n'est nullement (cf. la remarquable analyse de mcCoy 13 passim). Le message principal du texte est que toute participation, si minime soit-elle, à l'économie de marché était politiquement dangereuse. Implicitement, Jefferson adoptait une 
conception de l'économie que l'on pourrait presque qualifier de proto-marxiste. En effet, l'échange marchand était nécessairement inégal à ses yeux ; l'acte de vente introduisait une « dépendance » qui pouvait être exploitée à des fins politiques, par des ambitieux, ce qui implique que les « clients» du texte n'étaient pas d'humbles citoyens, mais avant tout des marchands, de "gros» clients, capables d'envisager la reconstitution d'une oligarchie aux États-Unis, et que les interlocuteurs de ces " clients " à l'inverse étaient bien des petits producteurs en situation subordonnéee, artisans surtout puisque les agriculteurs étaient censés échapper à ce sort funeste ${ }^{5}$. Encore une fois, il ne s'agissait pas pour autant de promouvoir une autarcie impossible. Mais, et ce sera un souci constant chez Jefferson, il fallait, malgré les risques posés par cet échange inégal, parvenir à garantir le possibilité d'une indépendance économique de chaque producteur/électeur par rapport aux jeux du marché et à ses maîtres, les marchands.

4 Cette description de la société comme menacée par l'hégémonie économique des moneyed men n'est sans doute pas suffisamment prise à la lettre dans l'analyse des débats politiques de la Jeune République. Pourtant, elle correspond à une réalité profonde et observable, la position dominante - il serait possible de parler de « classe dominante »-des possesseurs d'un accès privilégié au marché dans le jeu économique de l'époque, par rapport à une masse de producteurs indépendants d'une part, de personnes dépendantes de ces producteurs d'autre part (femmes mariées, esclaves, etc.) dont le rapport au marché était aussi variable que les bénéfices qu'ils en tiraient (cf. Gervais 1995 et 1996). L'opposition entre husbandmen et mechanics introduite par Jefferson dans son texte recouvrait une distinction plus fine entre producteurs intégrés dans une économie marchande au point d'être dépendants de ceux qui leur assuraient l'accès à cette économie, et producteurs capables de se passer des services de ces mêmes intermédiaires. Ainsi, il est significatif que Jefferson identifie plus loin dans le même passage les artisans dépendants aux artisans urbains. En milieu rural, les artisans regagnaient leur indépendance, parce qu'ils ne dépendaient pas de marchands, mais d'une clientèle agricole multiple et modeste avec laquelle ils pouvaient rester solidaires politiquement. De même, la phrase n'interdit pas d'envisager l'existence d'agriculteurs corrompus, car spécialisés au point de dépendre pour leur subsistance des caprices du marché. Le problème n'était donc pas l'échange commercial en soi (un fait de nature pour tous les penseurs du xVIII ${ }^{e}$ siècle), mais l'échange commercial en tant qu'il pouvait conduire à une domination politique de ses principaux bénéficiaires, les marchands.

5 Il s'agissait donc de parer le danger d'une utilisation politique d'une situation de domination économique que Jefferson considérait d'une manière implicite comme potentiellement inscrite dans les rapports économiques de son temps. La "classe dominante " qui contrôlait le marché, ses mécanismes, et en tirait l'essentiel des bénéfices ${ }^{6}$ cherchait à maximiser le nombre de ses clients parmi les producteurs indépendants, en développant les activités marchandes. Ceux qui cédaient aux sirènes de l'échange marchand se retrouvaient peu à peu dépendants - et constituaient une masse de manœuvre politiquement utilisable, dans un objectif de conquête total de la société, à une époque où le vote à bulletin secret n'existait évidemment pas ${ }^{7}$. L'ensemble du programme fédéraliste constituait aux yeux de Jefferson une machine de guerre destinée à priver de leur indépendance-d'abord économique, puis politique - ceux des producteurs indépendants qui ne faisaient pas déjà partie de la clientèle marchande. Notons au passage qu'il ne s'agissait pas d'une analyse vulgaire du 
politique en termes d'intérêts personnels; pour Jefferson, il existait des fédéralistes sincères, mais égarés, comme John Adams, qui mésestimaient le danger de dictature de la classe économiquement dominante et surestimaient les risques de révolution sociale venant de la masse de la population. Cette erreur d'analyse conduisait ces hommes à vouloir protéger une classe sociale qui n'avait nul besoin de protection, mais qui devait être au contraire collectivement et étroitement surveillée et limitée dans son pouvoir économique et politique.

6 Même les positions internationales de Jefferson découlaient de la même nécessité de maximiser l'indépendance des producteurs par rapport aux marchands (Hendrickson et Tucker, Rossignol). Ces derniers étaient certes un mal nécessaire (pas plus que Rousseau, Jefferson n'envisageait un retour à l'état de nature, et bien plus que Rousseau, il admettait pragmatiquement le caractère inévitable de la division de la société en classes, marchands / dépendants des marchands / indépendants des marchands). Mais ce mal devait être circonscrit politiquement ; et, au début des années 1780, si l'on suit les Notes, Jefferson espérait, semble-t-il, garder autant que possible les marchands et leurs affidés à l'étranger. Mieux valait perdre le bénéfice du commerce sur l'Atlantique, que de voir se développer une puissante classe marchande américaine ; mieux valait acheter à prix d'or les produits manufacturés en Angleterre que de voir apparaître aux États-Unis des masses d'électeurs urbains entièrement inféodés aux marchands qui les employaient, et menaçant de ce fait la république. Par ailleurs, l'expansion vers l'Ouest, en autorisant le développement de la population des fermiers indépendants, était une nécessité politique beaucoup plus qu'économique ; là encore, il s'agissait de modifier un rapport de forces autant que possible en faveur d'activités non marchandes, ou le moins marchandes possible. Même l'ambiguïté de Jefferson par rapport à l'esclavage mériterait d'être revue à la lumière de cette position trop souvent résumée comme agrarienne, et qui serait plutôt anti-marchande ; mais ce point complexe mériterait une étude propre, et ne sera pas abordé ici.

7 Enfin, pour compléter ce bref tableau, notons que pour Jefferson comme pour la grande majorité des observateurs politiques de son temps, la vertu - identifiée dans le passage des Notes cité plus haut à la conscience du risque marchand - se trouvait certes en quantité limitée, mais partout dans la société, y compris chez des marchands, de même que l'on pouvait trouver des agriculteurs convaincus, par corruption ou aveuglement, de la nécessité de développer l'activité marchande. Il y avait cependant une réalité statistique ( "la masse des cultivateurs », comme dit le texte), liée au fait que l'intérêt personnel n'était pas systématiquement absent des calculs politiques des êtres humains. L'intérêt personnel du marchand le conduisait à vouloir corrompre la société et la république, celui de l'agriculteur indépendant le poussait à combattre cette corruption. En d'autres termes, les agriculteurs indépendants vertueux suivaient leur vertu, mais les agriculteurs moins vertueux étaient au moins susceptibles de suivre leur intérêt, qui les conduisait aussi (le plus souvent) à une position vertueuse. En revanche, un membre des moneyed classes ou des couches qui dépendaient des ces dernières ne pouvait être vertueux que contre son intérêt - ce qui le rendait certes deux fois plus méritant, mais impliquait aussi qu'une telle attitude ne serait adoptée que par des membres de ces couches déjà très vertueux au départ. L'attitude générale de méfiance à l'égard du commerce que cette analyse supposait resta une constante de la pensée de Jefferson, et se retrouve bien sûr dans son disours inaugural de 1801 - d'où est tiré notre titre ; elle est aussi au cœur de l'ensemble des réflexions de ceux qui le soutiennent, à commencer par Madison, qui reprend quasi textuellement dans l'un de 
ses articles de 1792 publié dans la National Gazette de Freneau le passage des Notes cité plus haut ${ }^{8}$.

8 Il n'est pas question de nier que Jefferson respectait la propriété privée, le libre jeu du marché, ou souhaitait le développement économique (encore que sur ce dernier point, comme l'a montré Drew McCoy, une certaine ambivalence devait régner, puisque tous les adeptes de la science politique de l'époque étaient convaincus que ce développement entraînerait à terme la corruption du système, et au minimum la chute de la République : cf. McCoy 1980 passim). Mais toutes ces positions étaient articulées autour d'une profonde méfiance à l'égard des couches supérieures du monde marchand, d'ailleurs éminemment liées à l'Angleterre encore dans les années 1780 . Même après le développement, dans les années 1790, d'intérêts commerciaux et manufacturiers opposés au maintien des États-Unis dans l'orbite britannique, intérêts d'ailleurs très souvent pro-jeffersoniens, Jefferson et d'autres restèrent très hostiles à toute concentration du pouvoir économique. Comment expliquer autrement l'échec final de la Première Banque des États-Unis, et l'hostilité forcenée de Jefferson aux banques $^{9}$ ? Tout ce qui pouvait ressembler à des pratiques monopolistiques, anti-concurrentielles, privilégiant certains acteurs économiques au détriment d'autres, devait être combattu, et ce n'était pas une clause de style dans un monde où ces pratiques constituaient le pain quotidien de la classe marchande. Les démocrates républicains acceptaient sans peine la nécessité d'une classe marchande aux États-Unis, et acceptaient même, après 1790, l'idée d'un développement commercial général, mais à condition que ces évolutions soient contrôlées de façon à éviter tout profit marchand excessif, et à en distribuer les fruits le plus largement possible. En somme, la défense par Jefferson de l'économie de marché libérale ressemble à ce que serait une défense du capitalisme industriel aujourd'hui articulée autour de l'objectif d'une limitation du profit des patrons par la protection systématique des intérêts des salariés. Certes, il n'y a pas là stricto sensu un discours révolutionnaire, mais il est n'est guère possible à mon sens d'y voir une défense et illustration enthousiaste du système libéral. Le contraste avec Hamilton est d'ailleurs éclairant.

B) Hamilton et l'encouragement du commerce

9 L'indépendance économique est décidément un terme clé entre tous dans le vocabulaire politique de la Jeune République. Car c'est au nom de la même volonté d'indépendance, élargie cette fois à la nation tout entière, qu'Hamilton pouvait prendre le contre-pied de Jefferson, dès 1791 , dans son «Rapport sur les manufactures ${ }^{10}$. Le supposé anglophile Secrétaire d'État au Trésor concevait explicitement le développement des manufactures, et surtout d'une classe marchande en bénéficiant, comme une machine de guerre dirigée contre la domination économique anglaise. Persuadé de l'existence de ce que l'on appelle aujourd'hui l'«échange inégal », il considérait l'approche jeffersonienne comme une dangereuse utopie, défendable en principe, mais pas dans un monde réel dominé par les politiques mercantilistes et les entorses au laissez-faire. En se méfiant trop des intérêts marchands, Jefferson mettait en danger la survie même de la république, que l'absence de développement finirait par faire retomber dans l'escarcelle des puissances aristocratiques européennes. Dans l'esprit de Hamilton, pour employer des termes anachroniques (mais qui n'en conviennent pas moins, aussi surprenant que cela puisse paraître), la concurrence économique entre les nations primait sur la lutte économique entre les classes à l'intérieur de chaque nation. Le Secrétaire au Trésor admettait parfaitement la division de la société en couches - ou classes - différentes, mais affirmait implicitement que 
les bénéfices tirés par tous les participants à un processus de développement marchand l'emportaient sans conteste sur d'éventuelles inégalités engendrées par l'échange marchand lui-même, inégalités qu'il n'évoque d'ailleurs à aucun moment dans son texte ${ }^{11}$.

Plus fondamentalement, Hamilton développait une théorie propre du fonctionnement économique, disjointe de l'analyse politique. Le jeu des intérêts privés, combiné à une intervention de l'État raisonnée et mesurée (Hamilton rejette explicitement le libre-échange et le laissez-faire smithien, comme autant d'utopies inapplicables au monde réel fait de gouvernements mercantilistes et d'agents économiques irrationnels), pouvait conduire à l'enrichissement général ; ne pas suivre cette route, c'était justement s'exposer à la catastrophe que Jefferson faisait mine de redouter, l'effondrement de la république. Certes, quelle que soit l'influence de Hume sur sa pensée, Hamilton ne niait pas l'existence d'hommes vertueux. Mais il estimait que leur travail politique ne pouvait être opératoire qu'à partir du moment où l'intérêt personnel des membres de la société, c'est-à-dire leur bien-être matériel, avait été pris en compte et assuré. Or ceci n'était possible que grâce au développement marchand. Même si les politiques plus ou moins mercantilistes des gouvernements français et britanniques n'avaient pas imposé une politique équivalente de la part du gouvernement des États-Unis pour compenser le handicap des producteurs américains à l'exportation, le développement marchand n'en aurait pas moins été une évolution hautement souhaitable, puisque bénéficiant à tous. Mais ce qui était simplement utile dans un monde idéal devenait indispensable dans le monde réel, où ne pas avancer, c'était reculer. Ne pas développer la puissance de la classe dirigeante marchande, seule aune véritable de la puissance d'un pays et d'un gouvernement aux yeux de Hamilton, c'était accepter la mort de la république, soit par mainmise de l'étranger, soit à la suite d'une révolution sociale intérieure provoquée par le désordre, la misère et le mécontentement. Asseoir le crédit des États-Unis, développer des manufactures au service des élites marchandes, fortifier ces couches supérieures de la classe marchande, éviter un affrontement économique direct avec une Angleterre économiquement surpuissante, profiter au contraire de l'éclipse française, maintenir à l'intérieur l'ordre et la sécurité des biens sans lesquels aucun développement économique n'était possible, tous ces objectifs ne constituaient en rien pour Hamilton des complots contre la république, mais étaient des conditions préalables à la survie de cette république : économie d'abord, politique ensuite. A terme, la constitution d'une élite marchande puissante, aussi puissante peut-être que l'élite anglaise, assurerait l'indépendance des États-Unis (et, là encore, amènerait sans doute la fin de la République, ou tout au moins une démocratie encore plus restreinte ; mais sur ce point le pessimisme de Hamilton était partagé par Madison et Jefferson, même si ces derniers pensaient pouvoir reculer l'échéance plus loin dans le temps).

11 Ce but ne valait bien sûr que parce que le développement de la puissance marchande avait dans l'ensemble des conséquences bénéfiques pour tous, ce qui nous ramène à l'idée centrale de notre analyse. Dans les économies politiques de Hamilton et de Jefferson, c'est bien l'économie qui constituait le point de départ, et plus précisément deux visions diamétralement opposées du développement de la puissance marchande. Celui-ci devait être étroitement contrôlé car il constituait une source potentielle d'injustice pour l'un, et être encouragé à tout prix car source de prospérité générale pour l'autre. Le rejet par Jefferson et ses alliés virginiens ou new-yorkais de mesures qui semblaient de simple bon sens aux yeux d'Hamilton ne pouvait s'expliquer pour ce 
dernier que par une volonté corruptrice des premiers, manifestant l'avidité et la jalousie de miséreux incapables de profiter normalement du bon fonctionnement social, et devenus bandits de grand chemin au cours de leur carrière politique. Hamilton estimait que la vertu consistait à ne pas s'opposer à son propre intérêt et à celui des autres, le tout identifié à une économie en bonne santé, et celle-ci à une classe marchande puissante. Le caractère en apparence évident de cet intérêt n'empêchait pourtant pas la floraison de comportements contraires parmi ses semblables (et pas seulement dans les couches dites «inférieures »; le Secrétaire au Trésor, depuis la guerre d'Indépendance, avait contracté un solide mépris pour les spéculateurs à courte vue qui peuplaient à ses yeux les moneyed classes (cf. Nelson 49). Paradoxalement, parce que le système économique lui-même était vertueux, mais ne débouchait pas automatiquement sur une république vertueuse, Hamilton était amené à conclure que l'être humain était rien moins que vertueux (et inversement, la foi de Jefferson en ce même être humain était quotidiennement revivifiée par l'observation qu'un fonctionnement économique fondamentalement producteur d'injustice ne conduisait pas automatiquement au désastre politique du despotisme).

Conclusion de la première partie

Les positions de Jefferson et Hamilton, si l'on accepte la reconstitution que j'en ai proposée, ne doivent pas pour autant être prises comme les seules possibles. D'autres visions politiques plus radicales existaient. Citons les variantes les plus importantes au début des années 1790 , lorsque ce conflit de principe se cristallisa en opposition de parti. Pour les plus extrêmes des opposants à l'élite marchande, seule une décentralisation totale et l'application de principes révolutionnaires rigoureux pouvaient brider cette dernière, dont il faudrait peut-être même se débarrasser, et qu'en tout cas il fallait priver de tout lieu de pouvoir (l'anti-fédéralisme extrême dégénérant en une sorte de proto-anarchisme, chaque Américain régnant en maître sur sa maisonnée et sous son figuier, pour reprendre le vocabulaire de l'époque). A l'opposé, certains futurs «hauts fédéralistes» considéraient plus ou moins ouvertement que l'être humain était décidément trop vicié pour se plier de lui-même à l'ordre naturel qui lui apporterait le bonheur, que la république était une utopie, et que seul un gouvernement fort, fondé sur le principe d'autorité et réservé à une élite vertueuse (pas nécessairement héréditaire ; les avis divergeaient), pouvait faire fonctionner correctement la société. Mais ce que je souhaite souligner, c'est qu'en 1790 déjà, toute "économie politique» s'articulait implicitement autour de visions divergentes des avantages et des risques du développement économique, et des mesures à prendre en conséquence, et qu'en général ces visions étaient d'abord une prise de position sur la question de l'activité marchande - et en dernière analyse, sur le rôle de la classe marchande en société, même si le terme de classe n'était pas celui employé à l'époque (on parlait plutôt d'interest). En d'autres termes, les discours politiques témoignaient d'une conscience aiguë des conflits d'intérêt économiques, et tenter de les analyser sans inclure ceux-ci, c'est leur faire perdre une grande partie de leur cohérence. Plus encore, c'est se priver d'un élément central permettant de comprendre comment ils emportaient l'adhésion de différents groupes, et pas seulement chez les politiciens professionnels. Car ce que l'on observe au sommet se retrouve, sinon à la base, du moins assez près de celle-ci, pour que l'on sorte nettement du cadre restreint des élites; c'est du moins ce que démontre l'étude des toasts politiques. 
2. Discours public et économie politiqueA) Journaux et esprit de parti au début de l'ère fédéraliste : l'exemple des toasts

Les deux analyses que nous venons d'esquisser s'appliquent à l'ensemble de la pensée de Jefferson et de Hamilton, et dans une large mesure à celles des dirigeants politiques qui les entouraient. Mais, et c'est ce sur quoi nous souhaitons surtout insister, elles se retrouvent dans les discours politiques les plus divers, tels qu'ils remplissaient les colonnes des journaux de la Jeune République. La naissance des "partis", sous une forme embryonnaire, est généralement datée des années 1791-1793, et des premiers grands affrontements entre Hamilton et Jefferson. Mais la lecture de la presse de l'époque démontre que les deux idéologies que ces dirigeants incarnaient étaient déjà pleinement organisées. La transition d'un débat politique argumentatif à un débat conflictuel prit effectivement quelques années; il est frappant de constater à quels points les principaux journaux existant depuis les années 1780 acceptaient dans leurs colonnes des discours parfaitement contradictoires, juxtaposés simultanément ${ }^{12}$. Ce modèle de libre débat entre républicains de bonne foi fit place progressivement à un affrontement organisé, dans lequel la coloration politique de chaque journal était à peu près unifiée, le discours adverse n'étant plus considéré comme légitime. Cette transition n'est pas encore accomplie en 1793, du moins pour les journaux favorables aux positions de Hamilton, incontestablement les plus nombreux. La presse proto-républicaine est plus unifiée dans son discours, mais aussi très minoritaire ; on sait que Jefferson fera venir Freneau à Philadelphie précisément pour pallier ce peu d'écho de ses thèses dans les «médias ", mais ledit Freneau meurt dans l'épidémie de choléra qui sévit à l'automne 1793 dans la capitale, et son journal, la National Gazette, disparaît avec lui. Le navire-amiral de la presse républicaine, l'Aurora, n'est lancé qu'en 1794. De même, à Boston, le Columbian Centinel, plutôt favorable aux futures thèses fédéralistes, domine nettement son concurrent républicain, l'Independent Chronicle, qui n'est d'ailleurs qu'hebdomadaire. Heureusement, en 1793 encore, presque tous les journaux impriment indifféremment des points de vue pro-Hamilton et pro-Jefferson, le journal de Freneau constituant la grande exception ${ }^{13}$.

De surcroît, ce qui est imprimé est quasi totalement politisé, assurant un matériau abondant. Pratiquement chaque élément constituant un journal pourrait donner lieu à une analyse d'économie politique; les articles d'opinion plus ou moins anonymes et signés à la romaine, bien sûr, le traitement et le choix des nouvelles de l'étranger, mais aussi les poésies comiques ou lyriques, les entrefilets des nouvelles locales, et même les avis de décès. L'intérêt particulier des toasts portés lors de diverses célébrations civiques et publiés dans la presse vient du fait qu'il reflète le discours politique le plus général que l'on puisse rencontrer dans ce type de sources. Certes, la majorité de ces toasts proviennent de notables, et d'activistes politiques. Mais il s'agit dans de nombreux cas de notabilités locales, éloignées des cercles du pouvoir, mais actives dans la société des Cincinnati ou l'ordre de Saint Tammany, dans la milice de leur État, dans les fêtes civiques. Quelques toasts reflètent même une toute petite notabilité "provinciale » : fêtes patriotiques de Cranbery, New York, ou de Newark, New Jersey, alors à peine plus que des villages. A tout le moins, ce genre de fêtes ou de dîners réunit les cadres politiques locaux, et non plus seulement les dirigeants, et dans beaucoup de cas (les fêtes du 4 juillet, par exemple), l'audience est plus large, et inclut une bonne partie de la population ${ }^{14}$. 
Que les paroles ainsi attribuées à tel ou tel orateur d'après-dîner aient été prononcées ou non importe d'ailleurs peu. L'essentiel est qu'elles n'aient pas été inventées de toute pièce par l'éditeur du journal en suivant ses propres convictions politiques, et il semble bien que ce n'est effectivement pas le cas. Les dates correspondent aux distances, les toasts varient énormément en nombre et en style, les préambules sont d'ailleurs souvent vagues, voire carrément imprécis. Telle série de toasts du 4 juillet est donnée sans lieu, d'autres prononcés à diverses occasions sont rapportés sans date, les assemblées concernées sont le plus souvent précisément décrites lorsqu'il s'agit du lieu d'édition du journal, mais disparaissent lorsque la source est lointaine. Paradoxalement, la qualité très inégale des attributions est un argument en faveur de l'authenticité de la source ; un faux serait plus soigné. Autre indication, les protestations épisodiques qui saluent un toast mal attribué. Ainsi, en janvier 1793, deux officiers de la société des Cincinnati de Philadelphie signent un entrefilet dans le journal local niant qu'un dîner signalé dans une édition précédente ait été tenu en l'honneur de l'anniversaire de Washington. Début juin de la même année, toujours à Philadelphie, la publication des toasts d'un dîner au Belvedere Club de New York, tenu en l'honneur de l'anniversaire de George III, provoque une protestation indignée du secrétaire dudit club, qui n'aurait fait que concéder une salle à un groupe d'expatriés anglais. Tout ceci confirme une intervention éditoriale des plus restreintes, hormis le choix de la célébration elle-même. Ce qui est d'ailleurs logique si l'on se rapporte à la façon dont les journaux de l'époque sont construits, à grand renfort de pièces rapportées, articles du journal voisin aussi bien qu'extraits de journaux étrangers parfois vieux de plusieurs mois, contributions de notables locaux ou de dirigeants politiques en vue (presque toujours anonymes), entrefilets rajoutés au dernier moment pour boucher un trou, extraits de lettres d'amis ou de connaissances parfois fort indirectes, insérées au seul motif qu'elles se font l'écho d'événement lointain... De toute évidence, il n'est pas question de vérifier des sources!

Qui, alors, est à l'origine de ces toasts? Sans doute les mêmes notables locaux qui abreuvent les éditeurs de journaux de leur prose, de leurs poèmes plus ou moins bien venus, et communiquent ces extraits des lettres de leurs connaissances à l'étranger, que nous venons d'évoquer. Ils réarrangent sans doute les paroles des orateurs, en une série de déclamations à la fois emphatiques et concises, véritables exercices de style sur un canevas fixe. Entre 10 et 15 toasts en moyenne, pas plus d'une phrase ou deux, et des topoi obligés, tels la Constitution, les héros de 1776 ou «la beauté américaine ». Mais cette formalisation même est avantageuse, puisqu'elle met en valeur des variations aisément décelables, et remarquablement uniformes. Compte tenu de notre point de départ, il va de soi que nous avons choisi de comparer plus particulièrement un topos qui se retrouve dans la majorité des séries publiées, et que nous pourrions baptiser «toast économique ». Arrivant généralement assez tard dans la série (jamais dans les trois premiers toasts, rarement dans les trois derniers), il évoque, schématiquement, l'Américain au travail, les "classes laborieuses », en somme. Mais il n'apparaît pas partout systématiquement, ni de la même manière, dans les fêtes civiques, et ces variations (et ces absences) sont en elle-mêmes instructives.

B) Un échantillon préliminaire : les toasts de la Federal Gazette et de la Boston Independent Chronicle

17 L'échantillonnage de toasts présenté ici est encore extrêmement restreint ; il découle d'un projet plus important, actuellement mené en collaboration avec Alan Potofsky, et 
visant à analyser les différentes expressions d'économie politique présentes dans une série de journaux américains pendant l'année 1793, année de consolidation de l'« esprit de parti » et d'ouverture du débat sur la Révolution française. L'étude est en cours, et il ne s'agit donc ici que de résultats partiels. A ce stade, j'ai pu dépouiller deux journaux : la Federal Gazette de Philadelphie, et l'Independent Chronicle de Boston, pour toute l'année 1793. La Federal Gazette, qui apparaît de plus en plus hamiltonienne à mesure que l'année s'écoule, n'a cependant pas encore de ligne éditoriale parfaitement cohérente ; par exemple, elle publie de loin en loin de vigoureuses attaques contre les projets de Hamilton ou la politique de neutralité, et des défenses et illustrations de Jefferson ou de la Révolution française. Ceci dit, la majorité des articles d'opinion publiés sont nettement favorables à Washington, à la proclamation de neutralité (le Pacificus de Hamilton est repris dans la Gazette), et hostiles aux « excès » de la Révolution française. Il faudra donc bien sûr effectuer d'autres dépouillements, de la National Gazette de Freneau peut-être, ou mieux encore d'un journal local d'une région pénétrée par les idées jeffersoniennes, campagnes virginiennes ou villes de la frontière, par exemple. Notons cependant que la Federal Gazette était clairement un quotidien de second rang à Philadelphie par rapport à la Gazette of the United States de John Fenno, beaucoup plus nettement liée à Hamilton. Ceci explique peut-être son caractère relativement œcuménique, qui nous permet malgré tout d'obtenir quelques indices sur les toasts "proto-républicains ", et donc de consolider nos résultats en ce qui concerne les toasts "proto-fédéralistes", nettement dominants ici. L'Independent Chronicle est beaucoup moins riche : pro-jeffersonienne et "démocrate républicaine » dès le printemps 1793 , elle ne paraît qu'hebdomadairement, et ne publie que douze séries originales de toasts pour l'année, dont dix en janvier ; nous reviendrons sur ce point infra ${ }^{15}$.

Premier enseignement, le toast « économique » est souvent absent des célébrations liées aux « affaires étrangères » (vingt-sept en tout), c'est-à-dire en général à la Révolution française (vingt-cinq cas). En 1793, de telles réunions en l'honneur du mouvement français sont encore nombreuses (réceptions en l'honneur de l'ambassadeur Genêt, ou de tel capitaine de vaisseau français dans les grandes villes portuaires ; fêtes de commémoration du 10 août, ou plus rarement du 14 juillet ; réunions d'amicales de sympathisants de la France, autour d'expatriés français). Or seuls sept de ces vingt-sept toasts « étrangers » recensés ici incluent une référence à l'économie, contre onze des treize toasts " américains » (anniversaire de Washington et 4 juillet essentiellement). Il s'agit donc bien d'un élément interne aux États-Unis, élément controversé qui plus est, puisqu'il est passé sous silence lorsque des étrangers sont présents. Les différences régionales sont d'ailleurs intéressantes de ce point de vue. Quinze des vingt-huit séries de toasts publiées en 1793 dans la Gazette concernent soit des événements ou des représentants français (treize cas), ou une assemblée française fêtant un événement américain (un cas), outre deux cas particuliers (le dîner en l'honneur de George III déjà cité, et un dîner irlandais de la Saint-Patrick). Deux de ces quinze cas seulement incluent un "toast économique ", contre onze des treize séries liées à des événements proprement américains. A Boston, la situation se présente différemment. L'Independent Chronicle publie fin janvier pas moins de dix séries de toasts provenant d'un vaste ensemble de "fêtes civiques» en l'honneur des victoires françaises, données dans et autour de Boston, cinq de ces dix séries incluant un toast économique. Puis, en onze mois, l'on ne découvre plus que cinq autres publications de ce type, toutes entre mai et août, toutes pour la Révolution française, et encore trois d'entre elles sont recopiées de la Federal Gazette ou d'un autre journal de Philadelphie. Au total, l'Independent Chronicle 
jeffersonien ne publie aucun toast lié à une fête américaine ; l'absence des fêtes du 4 juillet est particulièrement surprenante.

19 Ces différences peuvent peut-être trouver un début d'explication grâce à l'analyse du contenu de ces «toasts économiques ». Ils se présentent en effet sous plusieurs formes. Premier cas, le plus courant (six séries sur treize à Philadelphie, trois sur cinq à Boston), un toast général au commerce et à d'autres branches économiques, construit de façon à les unifier sans hiérarchisation particulière, généralement en une seule formulation (dans un seul cas, " agriculture et commerce " puis « science et arts utiles " donnent lieu à deux toasts différents). Outre les déclarations brèves, alliant simplement "agriculture, commerce et manufacture ", par exemple, on trouve des discours plus développés : "que chaque branche fasse progresser les autres, et que toutes contribuent au bien-être public "; "que leur influence bénéfique calme la rage de guerre, de domination et d'injustice ", etc. Mais alors que la formule traditionnelle à Philadelphie évoque « l'agriculture, le commerce et les manufactures », l'agriculture est omise à Boston dans deux cas sur trois, au profit de la navigation, de la littérature, ou de références aux impôts.

Deuxième cas de figure, une position privilégiée du commerce ; mais il faut distinguer de quel commerce l'on parle, intérieur ou extérieur ${ }^{16}$. Dans deux séries de la Federal Gazette, le «travail » de l'agriculteur et de l'artisan est combiné à l'« entreprise » ou à la " prospérité » du marchand, ce qui entraine des implications intéressantes quant au caractère subordonné des deux premières classes. Une troisième série de même origine privilégie aussi clairement le commerce intérieur, en complétant le «toast économique " d'un deuxième toast "à l'amélioration des routes et des canaux ». A l'opposé, deux séries de Philadelphie, et deux de Boston, restreignent explicitement ou implicitement leur objet au commerce extérieur, en faisant référence aux «mers les plus éloignées ", à la « liberté du commerce », ou à son influence pacificatrice dans les rapports entre nations (le quatrième cas est plus ambigu, puisqu'il s'agit du dîner en l'honneur de Georges III, incluant un toast "à la prospérité et au commerce des États-Unis et de la Grande-Bretagne, et à leurs intérêts réciproques »; il ne s'agit pas forcément de commerce au sens économique du terme).

21 Enfin, il faut distinguer deux dernières séries, publiées après les cérémonies du 4 juillet dans la région de Philadelphie et à Pittsburgh. "Que l'agriculture, les arts et la science marchent main dans la main dans notre pays ", proclament les patriotes des Delaware Works (probablement un site industriel en Pennsylvanie orientale, mais l'absence d'indication plus précise ne permet pas de localiser ce site, ni de préciser son activité, sidérurgie, poterie, briques ou textile ?) ; et à Pittsburgh, un toast est porté «au commerce étranger et à la manufacture domestique ", le toast suivant saluant «l'agriculture et l'artisanat ». Ignoré par les artisans des Delaware Works, le commerce est rejeté hors du pays à Pittsburgh. Le contraste est net, et les lieux d'origine (un site industriel, et une ville de l'Ouest) correspondent de manière plausible à des régions à sympathie jeffersonienne plus marquée qu'ailleurs.

Ces nuances ont-elles un sens politique? En tout cas leur coïncidence avec ce qui me semble être au cœur du débat entre Jefferson et Hamilton est nette. Les toasts non hiérarchisés ne dénotent aucune méfiance particulière à l'égard du commerce, mis en valeur même dans certains cas. Les toasts portant sur le commerce étranger peuvent avoir deux sens bien différents, selon qu'il s'agit d'un simple souci local de grand port (c'est probablement le cas à Boston) ou d'une référence excluant les réseaux 
commerciaux intérieurs. Il est à noter que le toast appelant au développement du commerce extérieur américain sur «des mers lointaines » (donc contre les anglais, et sans les anglais) vient de la Société Tammany de New York, qui n'est peut-être pas encore le bastion démocrate qu'elle sera sous Aaron Burr, mais qui n'est pas non plus nécessairement très proche de Hamilton. Le cas du dîner de George III est particulier ; il peut s'agir aussi bien d'une authentique déclaration hamiltonienne défendant le commerce avec l'ancienne métropole que d'un faux jeffersonien attirant l'attention sur cet aspect du programme des «anglophiles». Enfin, les toasts des Delaware Works et de Pittsburgh sont difficiles à analyser autrement que comme l'expression d'une franche hostilité à la classe marchande américaine, dont les activités intérieures sont soigneusement omises. En tout état de cause, il existe bien deux pôles opposé, l'un pro-commercial explicitement ou par implication, l'autre beaucoup plus méfiant, voire franchement anti-commercial, et souvent lié (moins paradoxalement qu'il pourrait y paraître) au souci de développer un commerce extérieur libéré de la tutelle anglaise.

Ce dernier type de toast « jeffersonien » est certes encore rare dans notre échantillon. Les choix éditoriaux de journaux dont l'écrasante majorité au début de 1793 penche plutôt pour Hamilton ont pu nous priver de nombre d'entre eux. De surcroît, les futures élites jeffersoniennes sont encore en gestation, voire inexistantes dans certaines régions. Ceci explique peut-être l'attitude de l'Independent Chronicle, qui ne publie de toasts locaux qu'en liaison avec la Révolution française. Peut-être les toasts économiques prononcés étaient-ils un peu trop « hamiltoniens » au goût de l'éditeur, qui se faisait encore violence au début 1793, lorsqu'il s'agissait de la noble cause de la Révolution française, mais ne put se résoudre à ouvrir ses colonnes aux Hancock et aux Adams au moment du 4 juillet. De fait, l'absence de l'agriculture dans certains toasts ne correspond certes pas à la vulgate démocrate-républicaine. Choix politique délibéré face à l'absence d'enracinement jeffersonien dans une région de commerce et d'industrie, ou prudence d'un éditeur peu désireux d'aliéner sa clientèle, il faudra vérifier cette hypothèse en comparant les séries obtenues ici au contenu du Boston Centinel hamiltonien, une fois ce dernier dépouillé. Et l'on mesure le caractère précieux de la relative ouverture d'esprit d'Andrew Brown, l'éditeur de la Federal Gazette, qui sauve ainsi de l'oubli une vision de l'économie politique de toute évidence existant ici et là, et au-delà des cercles dirigeants du futur parti républicain. Cette vision deviendra monnaie courante par la suite, grâce à l'essor des journaux d'opposition à partir de 1794 ; citons par exemple, un peu plus au nord, à Trenton dans le New Jersey, et un peu plus tard, en 1801, ce magnifique exemple : «au fermier américain, que la prospérité accompagne sa charrue, et que la misère frappe ceux qui ont honte de la prendre en main ». Ou, toujours en 1801, à Hartford (Connecticut) : «à l'agriculture, l'activité la plus utile à l'homme, et la plus bénéfique à la liberté »; "au commerce, fait pour l'homme, et non l'homme fait pour le commerce ". C'est bien sûr le modèle de l'adresse inaugurale déjà citée supra, le commerce devenant « servante » d'activités plus nobles ${ }^{17}$. Conclusion générale provisoire

24 Le «toast économique » a ce double avantage qu'il exprime une gamme de positions déjà bien différenciées sur l'économie politique du pays, et que ces positions ne sont pas celles de dirigeants politiques comme Hamilton, Jefferson, Madison ou d'activistes de premier plan comme Freneau. Il démontre l'existence d'une pensée politique de l'économie, plus répandue que l'on pourrait le croire, et avec laquelle le travail des grands noms que nous venons de citer entre en résonnance (ou en opposition). Pour le dire autrement, la pensée politique de la masse accompagne indépendamment (sinon 
précède !) celle des cadres dirigeants. Par ailleurs, cette pensée, quoique allusive, s'articule nettement autour de la question de la domination d'une classe particulière, celle des marchands, en prenant position pour ou contre l'extension de l'activité qui leur est propre, le commerce, à l'intérieur du territoire national, avec des conséquences directes sur le type de commerce extérieur considéré comme souhaitable. Si l'on admet que la société américaine de 1793 est bien une société articulée autour du commerce et d'un conflit potentiel d'intérêts entre ceux qui en profitent et ceux qui peuvent y perdre, alors le discours politique de l'époque devient véritablement un discours sur la façon de gérer les conflits sociaux - les conflits de classe, aurions-nous envie de dire ; en faisant confiance à la classe dirigeante, ou au contraire en s'opposant à son magistère. Le choix entre ces deux voies, soit dit en passant, n'est nullement déterminé par les positions socio-économiques des acteurs; il s'agit avant tout d'un pari sur l'avenir, étant entendu que personne ne souhaite vraiment «casser la machine " par une révolution sociale totale, et donc que tout le monde - ou presque - accepte les grands principes de l'économie libérale. Le tout est de savoir comment les appliquer, et sur ce point il me semble que les différences entre partis ont été très largement sous-estimées. L'accord apparent sur les principes du développement économique a trop fait oublier le fait que cette notion de « développement » n'était pas plus univoque alors qu'aujourd'hui, et que les discours sur les politiques économiques intégraient systématiquement une vision de la répartition des profits de ce développement. C'est en replaçant au centre du débat cette dernière question qu'il me parait possible de réconcilier les différents points de vue jeffersoniens, et de comprendre ce qui les séparait de ceux de leurs adversaires hamiltoniens. Le libéralisme de l'école d'Appleby porterait ainsi sur le fonctionnement général de l'économie, le républicanisme de Banning sur les rapports de force que ce fonctionnement suppose ; une hypothèse qu'il faudrait bien sûr étayer plus solidement que nous ne l'avons fait ici. Mais quel que soit le dosage correct entre les deux approches, il est important de partir de la réalisation que la question politique («qui va diriger?») recouvrait et élargissait une question économique ("qui va profiter ?»), conditionnant fortement le «consensus libéral » apparent sur les structures économiques ("comment fonctionner?»). Admettre ce caractère conditionnel $\mathrm{du}$ consensus est absolument essentiel à mes yeux pour comprendre les luttes politiques de l'époque. Car de toute évidence la question était loin d'être sans importance aux yeux de tous ces notables locaux qui exprimaient leur propre vision en ce domaine à chaque banquet civique.

\section{BIBLIOGRAPHIE}

Appleby, Joyce. Capitalism and a New Social Order : The Republican Vision of the 1790s. New York : New York UP, 1984.

Appleby, Joyce. « Jefferson and his Complex Legacy ». Jeffersonian Legacies. Ed. Peter S. Onuf. Charlottesville : UP Virginia, 1993. 1-16.

Banning, Lance. The Jeffersonian Persuasion : Evolution of a Party Ideology. Ithaca : Cornell UP, 1978. 
Cohen, I. Bernard. Science and the Founding Fathers : Science in the Political Thought of Jefferson, Franklin, Adams and Madison. New York : Norton, 1995.

Eicholz, Hans L. Harmonizing Sentiments : the Declaration of Independence and the Jeffersonian Idea of Self-Government. New York : Lang, 2001.

Elkins, Stanley et Eric McKitrick. The Age of Federalism : The Early American Republic, 1788-1800. New York : Oxford UP, 1993.

Engeman, Thomas S. éd. Thomas Jefferson and the Politics of Nature, Notre Dame : UP Notre Dame, 2000.

Gervais, Pierre. « La révolution industrielle, progrès technique ou crise de l'économie de marché ?». RFEA 64 (1995) : 329-337.

Gervais, Pierre. « Gestion et profit marchands avant la "révolution industrielle" : l'exemple d'une route privée entre New York et Philadelphie, 1815-1828 ». Le Mouvement social 176 (1996) : 47-67.

Golden, Alan L. et James L. Golden. Thomas Jefferson and the Rhetoric of Virtue. Lanhal [Md.] : Rowman Littlefield, 2002.

Gordon-Reed, Annette. Thomas Jefferson and Sally Hemings : An American Controversy. Charlottesville : UP Virginia, 1997.

Hendrickson, David C. et Robert W. Tucker. Empire of Liberty: The Statecraft of Thomas Jefferson. New York : Oxford UP, 1990.

Horn, James, Jan Ellen Lewis et Peter S. Onuf, ed. The Revolution of 1800 : Democracy, Race, and the New Republic. Charlottesville : UP Virginia, 2002.

Lamoreaux, Naomi R. Insider Lending : Banks, Personal Connections, and Economic development in Industrial New England. New York : Cambridge UP, 1994.

Lathem, Edward C. éd. Chronological Tables of American Newspapers, 1690-1820. Barre [Mass.] : American Antiquarian Sty. \& Barre Publ., 1972.

Lienesch, Michael. «Thomas Jefferson and the American Democratic Experience : The Origins of the Partisan Press, Popular Political Parties, and Public Opinion ». Jeffersonian Legacies. Ed. Peter S. Onuf. Charlottesville : UP Virginia, 1993. 316-339.

Malone, Dumas. Jefferson and his time. Boston : Little Brown, 1948-1981, 6 vols.

McCoy, Drew R. The Elusive Republic : Political Economy in Jeffersonian America. New York : Norton, 1980.

McDonald, Forrest . Alexander Hamilton : A Biography. New York : Norton, 1979.

Nelson, J. R., Liberty and Property : Political Economy and Policymaking in the New Nation, 1789-1812. Baltimore : Johns Hopkins UP, 1987.

O'Brien, Conor Cruise. The Long Affair : Thomas Jefferson and the French Revolution, 1785-1800. Chicago : UP Chicago, 1996.

Peterson, Merrill D. The Jefferson Image in the American Mind. New York : Oxford UP, 1960.

Peterson, Merrill D. Thomas Jefferson and the New Nation : a Biography. New York : Oxford UP, 1970.

Pocock, J[ohn] G[reville] A[gard]. The Machiavellian Moment : Florentine Political Thought and the Atlantic Republican Tradition. Princeton : Princeton UP, 1975.

Read, James H. Power Versus Liberty : Madison, Hamilton, Wilson, and Jefferson. Charlottesville : UP Virginia, 2000. 
Rodgers, Daniel T. « Republicanism : the Career of a Concept ». Journal of American History 79-1

(1992) : 11-38.

Rossignol, Marie-Jeanne. Le ferment nationaliste. Paris : Belin, 1994.

Schwarzlose, Richard A. Newspapers : A Reference Guide. New York : Greenwood, 1987.

Sheldon, Garret W. The Political Philosophy of Thomas Jefferson. Baltimore : Johns Hopkins UP, 1991.

Smith, James M. The Republic of Letters : the Correspondence between Thomas Jefferson and James

Madison, 1776-1826. New York : Norton, 1995.

Stewart, Donald H. The Opposition Press of the Federalist Period. Albany : SUNY UP, 1969.

Syrett, Harold C. éd. The Papers of Alexander Hamilton. New York : Columbia UP, 1961-1987, 27 vols.

Tise, Larry E. The American Counterrevolution : A Retreat From Liberty, 1783-1800. Mechanicsburg [Pa.] : Stackpole, 1998.

Waldstreicher, David. In the Midst of Perpetual Fetes : The Making of American Nationalism, 1776-1820. Chapel Hill : UP North Carolina, 1997.

Weisberger, Bernard A. America Afire. Jefferson, Adamas, and the Revolutionary Election of 1800. New York : Morrow, 2000.

Wood, Gordon S. The Radicalism of the American Revolution. New York : Knopf, 1992.

Zuckert, Michael P. Natural Rights Republic : Studies in the Foundation of the American Political Tradition. Notre Dame : UP Notre Dame, 1997.

\section{NOTES}

1. En une note, il est impossible de décrire, ou même de résumer l'historiographie jeffersonienne. Une bibliographie commentée à jour jusqu'en 1997 est disponible sur Internet à l'adresse suivante : http://etext.virginia.edu/jefferson/bibliog. Je me contenterai de renvoyer le lecteur aux deux travaux bibliographiques les plus généralement admis comme essentiels (Malone et Peterson 1970), et à quelques ouvrages qui peuvent servir d'introduction au débat sur la pensée des dirigeants politiques de la Jeune République. Pour la politique, cf. Pocock, Banning, Sheldon, Wood et McCoy. Le libéralisme économique des jeffersoniens a été réévalué grâce au travail refondateur de Appleby 1984 ; mais l'étude la plus précise des politiques économiques et de l'économie politique jeffersonienne est celle de Nelson. Depuis le début des années 1990, les débats sur l'économie politique jeffersonienne ont pratiquement disparu; le concept de « consensus libéral » forgé par Appleby et son école domine presque sans partage les visions de l'économie politique de la Jeune République. Les publications plus récentes concernant Jefferson en particulier se concentrent sur les problèmes d'esclavage (avec la controverse autour de Sally Hemmings, cf. entre autres Gordon-Reed), l'évaluation des différences entre Madison et Jefferson (Smith, Read), ou cherchent à mieux cerner les sources philosophiques (Cohen, Zuckert, et les articles dans Engeman), la logique théorique interne (Eicholz) ou les formes rhétoriques des principes jeffersoniens (Golden et Golden). Le terme même d'économie politique est de plus en plus rarement employé, d'ailleurs. A noter tout de même, bicentenaire oblige, quelques ouvrages sur l'élection de 1800 , que je n'ai malheureusement pas pu utiliser 
(Weisberger, Horn et al.), et deux pamphlets symétriques et peu convaincant, faisant de Jefferson soit un conservateur raciste (Tise), soit un révolutionnaire enragé (O'Brien). 2. Le primat du politique, explicite dans l'historiographie « républicaine », n'est pas moins important chez les tenants de l'explication « libérale »; Joyce Appleby elle-même considère que «l'héritage le plus solide de Jefferson [...] a été une vue particulière de la liberté personnelle », et que c'est cette vision progressiste, moderniste de la liberté qui l'opposait avant tout à ses adversaires hamiltoniens (Appleby 1993, surtout 3 et 5 passim). Le différend est bien politique et non économique. John R. Nelson est le seul auteur récent à ma connaissance à enraciner l'opposition entre Jefferson et Hamilton dans un conflit économique, mais il réduit ce conflit à une différence de tactique, à l'intérieur d'un projet global identique (des États-Unis indépendants, républicains et économiquement puissants) ; cf. Nelson 1987, en particulier 68-69.

3. Le caractère à la fois contradictoire et inattaquable des positions de Banning et Appleby est bien résumé dans Lienesch, qui souligne, comme Rodgers, que toute analyse cohérente de Jefferson doit intégrer à la fois le libéralisme économique et le souci républicain de communauté, pourtant contradictoires ; mais il n'offre aucune solution convaincante.

4. Thomas Jefferson. «Query XIX : The present state of manufactures, commerce, interior, and exterior trade? » Notes on the State of Virginia. New York : Harper \& Row, 1964157.

5. Il me paraît indiscutable que le groupe des « dépendants " attaqués ici est celui des artisans insérés dans l'échange marchand (manufacturers et mechanics ne pouvant se rapporter à des professions industrielles, ouvriers d'industrie ou patrons, qui n'existaient pas encore aux États-Unis en 1785, et à peine en Grande-Bretagne même !). Tout le reste du texte est consacré à ces artisans, aussi bien avant qu'après la phrase citée : ce sont leurs masses (les mobs) qui sont violemment dénoncées par Jefferson. Si les « dépendants » étaient des marchands obséquieux à l'égard de leurs clients, interprétation parfois proposée en France, il faudrait en conclure que ce sont ces clients, des artisans puisque les agriculteurs sont majoritairement exempts de vices, qui sont « ambitieux ». C'est contraire à la fois à la lettre du texte, qui dénonce plus loin, non l'ambition, mais bien le vice des masses urbaines, et à tout ce que l'on sait de la société américaine de la fin du XVIII ${ }^{\mathrm{e}}$ siècle. L'identification des « clients » comme marchands peut paraître surprenante aujourd'hui, mais c'est la seule façon de retrouver dans le texte la vision quasi universellement admise à l'époque d'une société se composant de trois groupes, ou interests :agricultural interest, manufacturing interest, et moneyed ou mercantile interest. Pour deux exemples très connus de cette description, $\mathrm{cf}$. Federalist $\mathrm{n}^{\circ} 10$ (Madison) et 35 (Hamilton), in The Federalist Papers (New York : New American Library, 1961).

6. D'où le fait que cette classe était aussi celle des moneyed men; collectivement, les maîtres du jeu marchands s'appropriaient l'essentiel du capital, une observation d'ailleurs confirmée dans les faits.

7. A noter que Hamilton lui-même est entièrement d'accord avec cette analyse ! Cf. Federalist $\mathrm{n}^{\circ}$ 35, loc. cit.

8. Cité dans Elkins 269. Comme la plupart des autres historiens de la période, Elkins et McKitrick privilégient l'analyse politique de l'opposition entre partis (aristocrates contre républicains, anglophiles contre francophiles), sans la relier fermement à l'idée de deux classes opposées par le fonctionnement économique, idée pourtant implicitement présente dans les passages de Madison qu'ils utilisent, et qui sont encore 
une fois remarquablement proches de l'argument des Notes utilisé ici, malgré les dix années qui séparent les deux textes.

9. C'est certainement le point le plus faible de Nelson. Cet auteur défend de manière très convaincante l'idée d'un triumvirat démocrate-républicain (Jefferson, Madison, Gallatin) fondamentalement favorable au développement économique, et particulièrement à l'expansion manufacturière (ce qui est logique à partir du moment où ces manufacturiers ne sont pas des industriels, mais des petits artisans organiquement liés à des couches rurales elles aussi en expansion). Mais le refus obstiné de ces mêmes dirigeants de créer des outils financiers de développement est incompréhensible dans ce cadre. Le rejet des banques se comprend beaucoup mieux si l'on admet que le « développement » proposé par Jefferson et ses alliés était soumis à certaines conditions socio-politiques. En d'autres termes, il était souhaitable si et seulement s'il se faisait sous le contrôle et au bénéfice des couches non marchandes de la société. Par définition, une banque excluait ces couches à l'époque : cf. Lamoreaux. 10. Hamilton n'a pas bénéficié des mêmes efforts historiographiques que Jefferson ; cf. surtout McDonald. L'analyse que John R. Nelson fait des politiques hamiltoniennes est de loin la plus convaincante (Nelson passim), et je m'en suis largement inspiré dans les paragraphes qui suivent pour ce qui est de leurs détails, mais les implications socio-économiques de ces politiques sont minorées. Là où Nelson voit surtout des problèmes techniques et tactiques, en particulier en matière fiscale, je serais porté à reconnaître des prises de position essentielles sur les ressorts internes et les conséquences souhaitables de tout développement économique.

11. Le "Rapport sur les Manufactures" est reproduit dans Syrett. Harold C. Syrett éd., The Papers of Alexander Hamilton, New York, Columbia University Press, 1961-1987, 27 vol. La vision socio-économique globale de Hamilton est très sensible dans Federalist $\mathrm{n}^{\circ}$ 35, loc. cit.

12. La meilleure introduction générale à la période se trouve dans Elkins. Il y a eu peu d'études historiques des journaux d'opinion en eux-mêmes, depuis Stewart. Cf.aussi Schwarzlose.

13. Une liste assez complète des journaux de l'époque est fournie dans Lathem. 14. A ma connaissance, le seul ouvrage évoquant, d'ailleurs assez indirectement, les toast utilisés ici est celui de Waldstreicher. Je n'ai pu trouver d'analyse de contenu de ces toasts dans l'historiographie de la Jeune République, mais mes recherches se poursuivent sur ce point.

15. Federal Gazette and Philadelphia Daily Advertiser, quotidien de Philadelphie, Andrew Brown éditeur et Boston Independent Chronicle and Universal Advertiser, hebdomadaire de Boston, Thomas Adams éditeur. Pour ne pas alourdir inutilement les notes, j'ai reporté en annexe le détail des toasts et leurs références. Les microfilms de ces journaux sont ceux de l'American Antiquarian Society ; je remercie ici Catherine Collomp et le CESAM, qui m'ont permis d'avoir accès à ces sources. J'ai supprimé les « doublons ", c'est-à-dire les séries de toasts publiées dans les deux journaux, de mes statistiques, en les attribuant au journal les ayant publié chronologiquement en premier. Cf. Annexe. 16. Je remercie Marie-Jeanne Rossignol, qui a attiré mon attention sur les problèmes posés par ces deux mots, et la nuance entre commerce intérieur et extérieur qu'ils pourraient recouvrir. Cette question est malheureusement insoluble dans le cadre d'un échantillon aussi restreint. En l'état, il semble bien que le mot commerce soit utilisé indifféremment pour le commerce intérieur et extérieur (cf. toasts $\mathrm{n}^{\circ} 8$ et 9 de la Boston 
Chronicle), alors que trade implique plutôt le foreign trade, mais ce dernier mot n'apparaît que trois fois, et uniquement à Boston.

17. Les deux toasts sont cités dans un hebdomadaire jeffersonien de Trenton (capitale du New Jersey), le Trenton True American, Matthias Day et Jacob Man éd., vol. 1. n 3, 24 mars 1801, en provenance de Bridgetown (New Jersey ?): « The American Farmer ; May Prosperity Attend the Plough, and Poverty to those who are Ashamed to Hold it $» \mathrm{~N}^{\circ} 4$, 31 mars 1801, en provenance de Hartford (Connecticut?) : « Agriculture : The Pursuit most Useful to Man, Most Friendly to Liberty ; Commerce : Made for Man, not Man made for Commerce ». Les deux séries proviennent de fêtes données à l'occasion de l'accès de Jefferson à la présidence, le 4 mars ou peu après.

\section{RÉSUMÉS}

This paper presents the preliminary results of a work in progress, the aim of which is an integration of Jeffersonian and Hamiltonian visions of political economy with the kind of public opinion statements which began to appear in newspapers after the end of the Constitutional debate, more specifically during the year 1793 . The working hypothesis used is that there was indeed around 1792-1793 a cristallization of two opposed worldviews, usually associated with Jefferson and Hamilton respectively, and built on conflicting views of the economy and its role, within and in spite of the « liberal consensus » stressed by recent historiography. This opposition was centered on contradictory assessments of the benefits and risks of merchant development, which can be observed easily in two important texts, the Notes on the State of Virginia and the Report on Manufactures. While Jefferson considered that the correlated growths of merchant power and market activity were inherently dangerous, and should be strictly controlled, Hamilton took a much more positive view of the same phenomena.

A second part relates these two positions to an as yet small sample of patriotic toasts given during the year 1793 and printed in Philadelphia and Boston papers. By carefully analyzing the use and wording of one particular kind of thematic toast, called « economic toast, » it can be shown that, within the discourse of local political actors, there were traces of the same differential view of merchant activity which structured national political discourse.

\section{AUTEUR}

\section{PIERRE GERVAIS}

Pierre Gervais est agrégé d'histoire, maître de conférences à l'Université Paris 8 - Vincennes-Saint Denis. Il a publié Les États-Unis de 1860 à nos jours (Hachette, « Les fondamentaux », 1998). 